\title{
TESTE DE CONDUTIVIDADE ELÉTRICA PARA SEMENTES DE FEIJÃO-MUNGO-VERDE ${ }^{1}$
}

\author{
ROBERTO FONTES ARAUJO², JOÃO BATISTA ZONTA ${ }^{3}$, EDUARDO FONTES ARAUJO4, \\ ELAINE HEBERLE ${ }^{3}$, FLÁVIA MIRANDA GARCIA ZONTA ${ }^{3}$
}

\begin{abstract}
RESUMO - A utilização de sementes de boa qualidade é fundamental para o estabelecimento adequado de uma lavoura. Para uma análise mais precisa da qualidade de sementes, faz-se necessário complementar as informações fornecidas pelo teste de germinação com testes de vigor, possibilitando, assim, selecionar os melhores lotes para comercialização e semeadura. Dentre esses testes, destaca-se o de condutividade elétrica. Objetivou-se neste trabalho estabelecer uma metodologia adequada para o teste de condutividade elétrica em sementes de feijão-mungoverde, considerando períodos de embebição e número de sementes. Foram utilizados seis lotes de sementes e o teste de condutividade elétrica foi realizado com as seguintes variações: 100, 75 e 50 sementes embebidas em $75 \mathrm{~mL}$ de água destilada a $25^{\circ} \mathrm{C}$, com leituras realizadas após 3 , $6,9,12$, e 15 horas de embebição. $\mathrm{O}$ teste de condutividade elétrica conduzido com quatro subamostras de 50 sementes embebidas em $75 \mathrm{~mL}$ de água destilada permite a separação dos lotes a partir de 3 horas de embebição, mostrando-se promissor na avaliação da qualidade das sementes de feijão-mungo-verde.
\end{abstract}

Termos para indexação: vigor, Vigna radiata (L.) R. Wilczek, teste de vigor.

\section{ELECTRICAL CONDUCTIVITY TEST FOR MUNG BEANS SEEDS}

\begin{abstract}
Good quality seeds are indispensable for successful crop establishment. A more precise analysis of seed quality requires a complementation of the information supplied by the germination with vigor tests to allow selection of the best lots for commercialization and sowing. Among these tests, the electric conductivity test is important. The objective of this study was to develop a suitable methodology for the electric conductivity test in mung bean seeds, with treatments of different imbibition periods and seed numbers. Six seed lots were used with the following treatments: 100,75 and 50 seeds imbibed in $75 \mathrm{~mL}$ of $25{ }^{\circ} \mathrm{C}$ distilled water, and evaluations after $3,6,9,12$, and 15 hours of imbibition. The electrical conductivity test done with four subsamples of 50 pure seeds soaked in $75 \mathrm{~mL}$ of distilled water at $25^{\circ} \mathrm{C}$, permits a separation of the lots after three hours of soaking, and this procedure would make a quality evaluation of mung bean seeds viable.
\end{abstract}

Index Terms: vigor, Vigna radiata (L.) R. Wilczek, vigor test.

${ }^{1}$ Submetido em 16/09/2009. Aceito para publicação em 07/11/2010.

${ }^{2}$ Eng. Agr. Dr. Pesquisador EPAMIG/CTZM, CEP 36570-000, ViçosaMG, e-mail: rfaraujo@ufv.br

${ }^{3}$ Eng. Agr., Dr., Analista B Embrapa Cocais, CEP 65055-310, São
Luís-MA, e-mail: jobazonta@hotmail.com, naniengel@yahoo.com.br, flaviaufv@gmail.com

${ }^{4}$ Eng. Agr. Dr. Professor Adjunto, Departamento de Fitotecnia, UFV, CEP 36570-000, Viçosa-MG, e-mail: efaraujo@ufv.br 


\section{INTRODUÇÃO}

O feijão-mungo-verde (Vigna radiata (L.) R. Wilczek) é importante leguminosa granífera muito cultivada na Ásia (Tickoo e Satyanarayana, 1998). Após a germinação de suas sementes são obtidos os brotos de feijão (moyashi), forma de consumo muito apreciada na China, Japão e EUA, dentre outros países. No Brasil, sua produção é incipiente, mas a tendência é crescente por causa do aumento da demanda pelo broto de feijão (Vieira et al., 2003).

A utilização de sementes de boa qualidade é fundamental para o estabelecimento adequado de uma lavoura. Para análise mais precisa da qualidade de sementes, faz-se necessário complementar as informações fornecidas pelo teste de germinação com testes de vigor, possibilitando, assim, selecionar os melhores lotes para comercialização e semeadura. A padronização dos testes de vigor é importante, pois à medida que as técnicas de manejo cultural tornam-se mais sofisticadas, aumenta a necessidade do uso de sementes de alta qualidade (McDonald, 1998). Dentre esses testes, destaca-se o de condutividade elétrica. Este se baseia na permeabilidade das membranas, avaliando características relacionadas à liberação de metabólitos durante a embebição das sementes (Barros e MarcosFilho, 1997).

A condutividade elétrica como teste de vigor é recomendado para sementes de ervilha e sugerido para soja (Association of Official Seed Analysts, 2002). No Brasil, é utilizado como teste de vigor com resultados promissores, principalmente para sementes de amendoim (Vanzolini e Nakagawa, 2005), soja (Dias e MarcosFilho, 1996), feijão caupi (Dutra et al., 2006) e algumas olerícolas como feijão-de-vagem e quiabo (Dias et al., 1998), abóbora (Vieira e Dutra, 2006) e pimentão (Panobianco e Marcos Filho, 1998; Oliveira e Novembre, 2005). Entretanto, existem poucos trabalhos com ênfase na condutividade elétrica para avaliação da qualidade fisiológica de sementes de feijão-mungo-verde, sendo encontrado na literatura somente trabalho realizado por Hampton et al. (1992).

A pesquisa tem demonstrado que vários fatores podem afetar os resultados do teste, tais como: idade da semente, genótipo, qualidade da água, temperatura, duração do período de embebição, grau de umidade e número de sementes (Vieira, 1994).

Para o número de sementes da amostra existem várias recomendações. Estudando o teste de condutividade elétrica em sementes de amendoim, Vanzolini e Nakagawa (2005) observaram que o teste de condutividade elétrica foi eficiente para classificar os lotes de sementes de amendoim em diferentes níveis de vigor, sendo a metodologia com quatro repetições de 50 sementes, embebidas a $25{ }^{\circ} \mathrm{C}$, a metodologia mais promissora. Loeffler et al. (1988) propuseram a utilização de quatro repetições de 50 sementes para a realização do teste, como forma de reduzir o coeficiente de variação. Alguns trabalhos, visando adequar o teste à determinada espécie, avaliaram diferentes números de sementes. Ribeiro et al. (1997) concluíram que para sementes de milho é possível detectar diferenças na qualidade fisiológica dos lotes com repetições de 25 sementes. Rodo et al. (1998) observaram em tomate que o teste de condutividade elétrica foi mais eficiente quando foram utilizadas 50 sementes para a cultivar IAC e 25 sementes para a cultivar Kada.

Quanto ao período de embebição, Vieira e Krzyzanowski (1999) citam que para sementes de ervilha e soja, o período de embebição deve ser em torno de 24 horas, pois durante este período ainda há lixiviação. Para sementes de feijão caupi cv. Setentão, Dutra et al. (2006) afirmam que o período de embebição das sementes deve ser 16 horas, sendo as sementes mantidas nas temperaturas de 25 ou $30^{\circ} \mathrm{C}$.

A maioria dos trabalhos com sementes de leguminosas como ervilha (Hampton e Tekrony, 1995) e soja (Tao, 1978; Loeffler et al., 1988; Marcos Filho et al., 1990; Krzyzanowski et al., 1991) recomendam o período de 24 horas de embebição para que se proceda a leitura da condutividade elétrica da solução de embebição. Entretanto, a rapidez na obtenção dos resultados é fator fundamental em programas de controle de qualidade de sementes (Dias e Marcos Filho, 1996). Neste sentido, alguns trabalhos têm mostrado a possibilidade de redução no período de embebição para avaliação da condutividade elétrica em soja (Marcos Filho et al., 1990; Dias e Marcos Filho, 1996) e em amendoim (Vanzolini e Nakagawa, 1997; Vanzolini e Nakagawa, 2005).

Devido à escassez de informações referentes à metodologia do teste de condutividade elétrica para sementes de feijão-mungo-verde, objetivou-se neste trabalho estabelecer metodologia para o teste de condutividade elétrica em sementes de feijão-mungoverde, considerando períodos de embebição e número de sementes. 


\section{MATERIAL E MÉTODOS}

O experimento foi conduzido no Laboratório de Pesquisa em Sementes, do Departamento de Fitotecnia, Universidade Federal de Viçosa-MG. Foram utilizados seis lotes de sementes de feijão-mungo-verde (Vigna radiata (L.) R. Wilczek), variedade Ouro Verde MG-2, provenientes da fazenda experimental da Empresa de Pesquisa Agropecuária de Minas Gerais. Os lotes foram formados por sementes produzidas nas safras 2006 e 2007, colhidos por diferentes métodos. Após a colheita, as sementes foram beneficiadas em máquina de ventilador e peneiras, o que foi eficiente para eliminação das impurezas e também das sementes de tamanho reduzido presentes nos lotes. Em seguida, os lotes foram classificados de acordo com a cor do tegumento. Não foi feita classificação por tamanho das sementes. Anteriormente a execução dos testes de qualidade, as sementes foram mantidas em câmara fria a $15^{\circ} \mathrm{C}$, em saco de pano. Assim, foram utilizados seis lotes: lote 1 (safra 2007/colheita única/ tegumento verde); lote 2 (safra 2007/colheita parcelada/ tegumento verde); lote 3 (safra 2007/colheita única/ tegumento marrom); lote 4 (safra 2007/colheita parcelada/ tegumento marrom); lote 5 (safra 2006/colheita única/ tegumento verde) e lote 6 (safra 2006/colheita única/ tegumento marrom).

Para a caracterização física e fisiológica dos lotes, as sementes foram submetidas aos seguintes testes:

Determinação do teor de água (\% b.u.): realizada utilizando-se o método da estufa, $105 \pm 3{ }^{\circ} \mathrm{C}$, durante 24 horas, com duas subamostras de 50 sementes, conforme especificações das Regras para Análise de Sementes (Brasil, 1992);

Germinação: foi realizado segundo as Regras para Análise de Sementes (Brasil, 1992), utilizando-se o rolo de papel germitest, temperatura de $25^{\circ} \mathrm{C}$ e quatro repetições de 50 sementes. As avaliações foram feitas no quinto e oitavo dias após a semeadura e o resultado expresso em porcentagem de plântulas normais;

Primeira contagem do teste de germinação: realizada conjuntamente com o teste de germinação, consistiu do registro das porcentagens de plântulas normais no quinto dia após a semeadura;

Envelhecimento acelerado: 200 sementes por repetição foram colocadas em caixas plásticas transparentes com tampa (gerbox), contendo $40 \mathrm{~mL}$ de água destilada. $\mathrm{O}$ material foi acondicionado a $42{ }^{\circ} \mathrm{C}$, durante 72 horas (Marcos Filho, 1999). Após esse período, quatro subamostras de 50 sementes por repetição foram submetidas ao teste de germinação descrito anteriormente;

Emergência de plântulas em campo: realizado com quatro repetições de 50 sementes, semeadas em sulcos com $3 \mathrm{~cm}$ de profundidade. A contagem foi realizada a partir da emergência da primeira plântula e prosseguiu até a estabilização. No momento da última contagem, determinou-se a porcentagem de emergência.

Condutividade elétrica: realizado com quatro repetições de 50, 75 e 100 sementes cada, pesadas e acondicionadas em copos de plástico descartáveis, com capacidade para $200 \mathrm{~mL}$, contendo $75 \mathrm{~mL}$ de água destilada. Em seguida, os copos permaneceram em câmara BOD, regulada a $25^{\circ} \mathrm{C}$, e a leitura foi realizada após $3,6,9,12$ e 15 horas de embebição, em condutivímetro (DIGIMED DM 31) sendo os resultados expressos em $\mu \mathrm{S} \mathrm{cm}^{-1} \mathrm{~g}^{-1}$.

Foi utilizado o delineamento inteiramente casualizado (DIC), com a comparação de médias pelo teste de Scott-Knott em $5 \%$ de probabilidade. Foi determinado o coeficiente de correlação de Pearson entre os valores obtidos no teste de condutividade elétrica e o teste de emergência em campo, separadamente para cada tratamento utilizado, sendo a significância dos valores de correlação determinada pelo teste $\mathrm{t}$ em $1 \%$ de probabilidade (Gomes, 2000).

\section{RESULTADOS E DISCUSSÃO}

Os dados referentes ao teor de água das sementes foram semelhantes entre os lotes estudados (Tabela 1). Esse fato é importante para a execução dos testes, pois a uniformidade do teor de água inicial das sementes contribui para obtenção de resultados consistentes (Loeffler et al., 1988). Verificou-se que o teor de água dos lotes estava em torno de $11 \%$ (b.u.), ou seja, dentro da faixa que não influencia nos resultados do teste de condutividade elétrica para a espécie (Hampton et al., (1992). De acordo com estes autores, o teor de água de sementes de feijão-mungoverde para realização do teste de condutividade elétrica deve estar entre 10 e $17 \%$.

A análise geral dos resultados dos testes de germinação, primeira contagem da germinação, envelhecimento acelerado e emergência em campo (Tabela 1) indicou que os lotes de sementes de feijão-mungo-verde utilizados no estudo apresentavam diferença em relação a sua qualidade fisiológica.

Pelo teste de germinação (Tabela 1), foi possível observar que os lotes 1, 2, 3, 4 e 5, apesar de haver diferença estatística entre eles, apresentavam porcentagem de 
germinação semelhante, sendo observados valores acima de $90 \%$ em todos os lotes. O lote 6 , com $66 \%$ de germinação, foi o que apresentou desempenho inferior aos demais. A semelhança no potencial germinativo entre os lotes é de fundamental importância em pesquisas com objetivo de determinação de metodologia para avaliação do vigor das sementes, pois o objetivo de um teste de vigor é separar lotes de sementes de germinação semelhante, porém com diferença quanto ao vigor. De acordo com Marcos Filho e Novembre (2009), a semelhança no potencial germinativo entre lotes de sementes é fator fundamental para determinação da diferença no vigor, pois se esses lotes apresentarem diferença muito acentuada na porcentagem de germinação o próprio teste de germinação, conduzido sob condições ótimas, consegue detectar diferença no potencial fisiológico das sementes.

TABELA 1. Resultados (\%) do teor de água (TA), germinação (G), primeira contagem de germinação (PC), envelhecimento acelerado (EA) e emergência em campo (EC) de seis lotes de sementes de feijão-mungoverde.

\begin{tabular}{cccccc}
\hline Lote & TA & G & PC & EA & EC \\
\hline 1 & 11,6 & $98 \mathrm{a}^{1}$ & $96 \mathrm{a}$ & $92 \mathrm{a}$ & $96 \mathrm{a}$ \\
2 & 11,5 & $95 \mathrm{a}$ & $93 \mathrm{a}$ & $79 \mathrm{~b}$ & $84 \mathrm{a}$ \\
3 & 10,7 & $92 \mathrm{~b}$ & $91 \mathrm{a}$ & $77 \mathrm{~b}$ & $85 \mathrm{a}$ \\
4 & 11,4 & $91 \mathrm{~b}$ & $90 \mathrm{a}$ & $63 \mathrm{~d}$ & $67 \mathrm{~b}$ \\
5 & 10,4 & $91 \mathrm{~b}$ & $88 \mathrm{a}$ & $71 \mathrm{c}$ & $85 \mathrm{a}$ \\
6 & 12,5 & $66 \mathrm{c}$ & $63 \mathrm{~b}$ & $60 \mathrm{~d}$ & $39 \mathrm{c}$ \\
\hline $\mathrm{CV}(\%)$ & - & 3,0 & 4,3 & 8,6 & 13,0 \\
\hline
\end{tabular}

${ }^{1}$ Médias seguidas pela mesma letra na coluna não diferem entre si pelo teste de Skott-Knott aos 5\% de probabilidade.

$\mathrm{Na}$ avaliação do vigor das sementes pela primeira contagem do teste de germinação (Tabela 1), não houve diferença entre os lotes com potencial germinativo acima de $90 \%$ (lotes 1, 2, 3, 4 e 5), sendo estes resultados obtidos em função das condições ótimas encontradas no teste de germinação. O lote 6 apresentou vigor inferior aos demais lotes quando avaliado pela primeira contagem do teste de germinação.

No teste de emergência em campo (Tabela 1), que é o teste que visa verificar o desempenho das sementes em condições de campo, os lotes 1, 2, 3 e 5 apresentaram porcentagem de emergência superior aos lotes 4 e 6 . Assim, considerando somente os lotes que apresentavam germinação acima de $90 \%$ (lotes 1, 2, 3, 4 e 5), nota-se a separação dos lotes quanto ao vigor, com os lotes 1,2 , 3 e 5 sendo classificados como de alto vigor e o lote 4 como de baixo vigor. O lote 6 , assim como na primeira contagem do teste de germinação, apresentou vigor inferior aos demais lotes.

No teste de envelhecimento acelerado (Tabela 1), pode-se observar maior estratificação dos lotes quanto ao vigor, o que sugere que este teste foi mais sensível para separar os lotes em níveis diferentes. Assim, o lote 1 foi classificado como de alto vigor, os lotes 2 e 3 como de vigor intermediário, o lote 5 como de baixo vigor e os lotes 4 e 6 como de menor vigor.

Analisando-se o vigor das sementes dos diferentes lotes (testes de primeira contagem de germinação, emergência em campo e envelhecimento acelerado), pode-se classificar o lote 1 como de maior vigor, o lote 6 como de menor vigor e os lotes 2, 3, 4 e 5 como de vigor intermediário. Pelos resultados do teste de condutividade elétrica, utilizando-se 100 sementes por repetição (Tabela 2), destaca-se a inferioridade no vigor dos lotes 4 e 6 e a superioridade no vigor do lote 1 , já na primeira leitura, realizada após 3 horas de embebição das sementes. Essa tendência foi observada até o período de embebição de 15 horas, destacando a eficiência do teste de condutividade elétrica em separar lotes com diferença de vigor, quando utilizadas 100 sementes por repetição. Os lotes 2,3 e 5 , assim como observado nos testes de emergência em campo e envelhecimento acelerado, apresentaram vigor intermediário. 


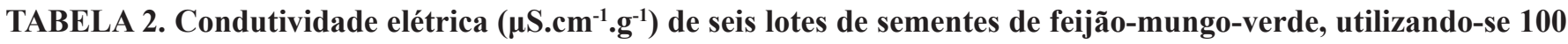
sementes embebidas em $75 \mathrm{~mL}$ de água destilada durante $3,6,9,12$ e 15 horas a $25{ }^{\circ} \mathrm{C}$.

\begin{tabular}{cccccc}
\hline \multirow{2}{*}{ Lote } & \multicolumn{5}{c}{ Período de embebição (horas) } \\
\cline { 2 - 5 } & 3 & 6 & 9 & 12 & 15 \\
\hline 1 & $20,6 \mathrm{a}^{1}$ & $64,7 \mathrm{a}$ & $85,1 \mathrm{a}$ & $94,7 \mathrm{a}$ & $96,4 \mathrm{a}$ \\
2 & $26,9 \mathrm{~b}$ & $65,5 \mathrm{a}$ & $87,1 \mathrm{a}$ & $91,6 \mathrm{a}$ & $92,8 \mathrm{a}$ \\
3 & $27,6 \mathrm{~b}$ & $71,8 \mathrm{a}$ & $94,2 \mathrm{~b}$ & $100,1 \mathrm{a}$ & $99,1 \mathrm{a}$ \\
4 & $42,7 \mathrm{c}$ & $86,3 \mathrm{~b}$ & $107,3 \mathrm{~d}$ & $116,7 \mathrm{c}$ & $118,4 \mathrm{~b}$ \\
5 & $19,5 \mathrm{a}$ & $69,6 \mathrm{a}$ & $99,3 \mathrm{c}$ & $107,5 \mathrm{~b}$ & $117,2 \mathrm{~b}$ \\
6 & $59,1 \mathrm{~d}$ & $101,4 \mathrm{c}$ & $132,5 \mathrm{e}$ & $146,9 \mathrm{~d}$ & $153,7 \mathrm{c}$ \\
\hline CV $(\%)$ & 11,6 & 6,1 & 4,5 & 4,5 & 5,6 \\
\hline
\end{tabular}

${ }^{1}$ Médias seguidas pela mesma letra na coluna não diferem entre si pelo teste de Skott-Knott aos 5\% de probabilidade.

De modo geral, o teste de condutividade elétrica realizado com 75 (Tabela 3) e com 50 sementes (Tabela 4) indicou, assim como no teste realizado com 100 sementes, maior vigor do lote 1 e menor vigor dos lotes 4 e 6 . Os lotes 2,3 e 5 , assim como observado nos testes de emergência em campo e envelhecimento acelerado, apresentaram vigor intermediário.

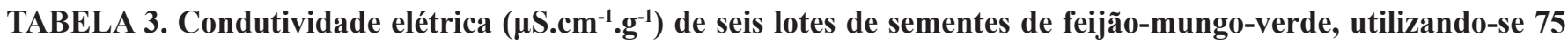
sementes embebidas em $75 \mathrm{~mL}$ de água destilada durante $3,6,9,12$ e 15 horas a $25^{\circ} \mathrm{C}$.

\begin{tabular}{cccccc}
\hline \multirow{2}{*}{ Lote } & \multicolumn{5}{c}{ Período de embebição (horas) } \\
\cline { 2 - 5 } & 3 & 6 & 9 & 12 & 15 \\
\hline 1 & $21,0 \mathrm{a}^{1}$ & $64,4 \mathrm{a}$ & $87,1 \mathrm{a}$ & $88,7 \mathrm{a}$ & $91,7 \mathrm{a}$ \\
2 & $29,4 \mathrm{~b}$ & $70,8 \mathrm{a}$ & $90,9 \mathrm{a}$ & $96,2 \mathrm{a}$ & $97,2 \mathrm{a}$ \\
3 & $30,0 \mathrm{~b}$ & $75,6 \mathrm{a}$ & $100,0 \mathrm{a}$ & $106,8 \mathrm{a}$ & $107,1 \mathrm{~b}$ \\
4 & $52,8 \mathrm{c}$ & $98,3 \mathrm{~b}$ & $116,2 \mathrm{~b}$ & $126,5 \mathrm{~b}$ & $127,8 \mathrm{c}$ \\
5 & $19,3 \mathrm{a}$ & $71,3 \mathrm{a}$ & $99,0 \mathrm{a}$ & $100,0 \mathrm{a}$ & $109,2 \mathrm{~b}$ \\
6 & $52,7 \mathrm{c}$ & $95,8 \mathrm{~b}$ & $121,7 \mathrm{~b}$ & $140,5 \mathrm{c}$ & $140,1 \mathrm{~d}$ \\
\hline CV $(\%)$ & 15,2 & 9,6 & 7,6 & 8,4 & 7,2 \\
\hline
\end{tabular}

${ }^{1}$ Médias seguidas pela mesma letra na coluna não diferem entre si pelo teste de Skott-Knott aos 5\% de probabilidade.

TABELA 4. Condutividade elétrica $\left(\mu \mathrm{S} . \mathrm{cm}^{-1} \cdot \mathrm{g}^{-1}\right)$ de seis lotes de sementes de feijão-mungo-verde, utilizando-se 50 sementes embebidas em $75 \mathrm{~mL}$ de água destilada durante $3,6,9,12$ e 15 horas a $25{ }^{\circ} \mathrm{C}$.

\begin{tabular}{cccccc}
\hline \multirow{2}{*}{ Lotes } & \multicolumn{5}{c}{ Período de embebição (horas) } \\
\cline { 2 - 5 } & 3 & 6 & 9 & 12 & 15 \\
\hline 1 & $22,9 \mathrm{a}^{1}$ & $60,1 \mathrm{a}$ & $82,5 \mathrm{a}$ & $88,4 \mathrm{a}$ & $90,8 \mathrm{a}$ \\
2 & $32,1 \mathrm{~b}$ & $74,0 \mathrm{~b}$ & $94,0 \mathrm{~b}$ & $100,5 \mathrm{a}$ & $101,1 \mathrm{a}$ \\
3 & $26,4 \mathrm{a}$ & $75,2 \mathrm{~b}$ & $104,9 \mathrm{c}$ & $111,6 \mathrm{~b}$ & $116,1 \mathrm{~b}$ \\
4 & $51,0 \mathrm{c}$ & $99,2 \mathrm{c}$ & $123,5 \mathrm{~d}$ & $134,6 \mathrm{c}$ & $138,2 \mathrm{c}$ \\
5 & $23,2 \mathrm{a}$ & $61,0 \mathrm{a}$ & $86,6 \mathrm{a}$ & $90,1 \mathrm{a}$ & $99,0 \mathrm{a}$ \\
6 & $49,9 \mathrm{c}$ & $97,6 \mathrm{c}$ & $126,6 \mathrm{~d}$ & $140,3 \mathrm{c}$ & $144,4 \mathrm{c}$ \\
\hline CV $(\%)$ & 11,3 & 6,9 & 6,2 & 6,8 & 7,3
\end{tabular}

${ }^{1}$ Médias seguidas pela mesma letra na coluna não diferem entre si pelo teste de Skott-Knott aos 5\% de probabilidade. 
Para confirmação dos resultados, foi calculado o coeficiente de correlação de Pearson entre o teste de condutividade elétrica e o de emergência em campo. A correlação dos dados obtidos em um teste de vigor com aqueles obtidos no teste de emergência em campo é de fundamental importância, pois de acordo com Marcos Filho (2009), para ser avaliado como eficiente, um teste de vigor deve proporcionar uma classificação dos lotes em diferentes níveis de vigor, de maneira proporcional à da emergência das plântulas (Tabela 5). Para todas as combinações de número de sementes e períodos de embebição, o coeficiente de correlação de Pearson foi significativo no nível de $1 \%$ de probabilidade. Os altos coeficientes de correlação encontrados entre os valores observados no teste de condutividade elétrica e no de emergência em campo corroboram com Vieira et al. (1999ab), que observaram relação entre os resultados do teste de condutividade elétrica e os de emergência de plântulas em campo para sementes de soja. A associação entre condutividade elétrica e emergência de plântulas em campo também foram observadas nos trabalhos de Oliveira et al. (1984) e Dias et al. (1996) com sementes de soja.

TABELA 5. Coeficiente de correlação de Pearson entre os valores do teste de condutividade elétrica, para todos os tratamentos, e os valores do teste de emergência em campo (EC), de seis lotes de sementes de feijãomungo-verde.

\begin{tabular}{|c|c|c|c|c|c|}
\hline \multirow{4}{*}{$\mathrm{EC}$} & \multicolumn{5}{|c|}{ Condutividade elétrica } \\
\hline & \multicolumn{5}{|c|}{100 sementes } \\
\hline & 3 horas & 6 horas & 9 horas & 12 horas & 15 horas \\
\hline & $-0.9713 * *$ & $-0.9752 * *$ & $-0.9611 * *$ & $-0.9575 * *$ & $-0.9231 * *$ \\
\hline & \multicolumn{5}{|c|}{75 sementes } \\
\hline \multirow{3}{*}{ EC } & 3 horas & 6 horas & 9 horas & 12 horas & 15 horas \\
\hline & $-0.8654 * *$ & $-0.8647 * *$ & $-0.9173 * *$ & $-0.9575 * *$ & $-0.9437 * *$ \\
\hline & \multicolumn{5}{|c|}{50 sementes } \\
\hline \multirow{2}{*}{$\mathrm{EC}$} & 3 horas & 6 horas & 9 horas & 12 horas & 15 horas \\
\hline & $-0.8622 * *$ & $-0.8482 * *$ & $-0.8736 * *$ & $-0.8874 * *$ & $-0.8981 * *$ \\
\hline
\end{tabular}

** Valores significativos no nível de $1 \%$ de probabilidade pelo teste $\mathrm{t}$.

No presente trabalho, a determinação do vigor das sementes pelo teste de condutividade elétrica foi coerente com os valores de vigor obtidos pelos testes de envelhecimento acelerado e emergência em campo, para todas as combinações de número de sementes por repetição e período de embebição. Assim, pode-se afirmar que o período de embebição de 3 horas, independentemente do número de sementes utilizadas por repetição, é eficiente para separação de lotes de sementes de feijão-mungoverde quanto ao vigor. O período de embebição de 3 horas representa uma significativa redução no período de condicionamento das sementes em relação ao período de 24 horas, adotado pela pesquisa como padrão para testes de condutividade elétrica para sementes de soja (Vieira e Krzyzanowski, 1999) e de ervilha (ISTA, 2004). Redução no período de embebição das sementes também foi observado com sementes de soja (Marcos Filho et al., 1990 e Dias e
Marcos Filho, 1995), de feijão-de-vagem e de quiabo (Dias et al., 1998), de tomate (Rodo et al., 1998), de brócolos (Martins et al., 2002), de berinjela (Novembre et al., 2002) e de amendoim (Vanzolini e Nakagawa, 1999ab).

Como um dos objetivos dos testes de laboratório é, além da redução do período necessário para condução do teste, reduzir a quantidade de sementes utilizadas, considerando-se os resultados observados nos testes de média e os coeficientes de correlação de Pearson, verificase que a utilização de 50 sementes por repetição foi a mais indicada no teste de condutividade elétrica para avaliação do vigor de sementes de feijão-mungo-verde.

\section{CONCLUSÃO}

O teste de condutividade elétrica, conduzido com quatro sub-amostras de 50 sementes puras embebidas em 
$75 \mathrm{~mL}$ de água destilada, permite a separação dos lotes a partir de 3 horas de embebição, mostrando-se promissor na avaliação da qualidade das sementes de feijão-mungoverde.

\section{AGRADECIMENTOS}

À Fundação de Amparo à Pesquisa do Estado de Minas Gerais (FAPEMIG) pelo financiamento do projeto.

\section{REFERÊNCIAS}

ASSOCIATION OF OFFICIAL SEED ANALYSTS. Seed vigor testing handbook. Lincoln: AOSA, 2002. 105p. (Contribution, 32).

BRASIL. Ministério da Agricultura e Reforma Agrária. Secretaria Nacional de Defesa Agropecuária. Departamento Nacional de Produção Vegetal. Coordenação de Laboratório Vegetal. Regras para Análise de Sementes. Brasília, DF, 1992. 365p.

BARROS, A.S.R.; MARCOS FILHO, J. Testes para avaliação rápida do vigor de sementes de soja. Revista Brasileira de Sementes, v.19, n.2, p.289-295, 1997.

DIAS, D.C.F.S.; MARCOS FILHO, J. Teste de vigor baseados na permeabilidade das membranas celulares: I. Condutividade elétrica. Informativo Abrates, v.5, n.1, p.26-36, 1995.

DIAS, D.C.F.S.; MARCOS FILHO, J. Testes de condutividade elétrica para avaliação do vigor de sementes de soja (Glycine max (L.) Merrill). Scientia Agrícola, v.53, n.1, p.31-42, 1996.

DIAS, D.C.F.S.; MARCOS FILHO, J.; CARMELLO, Q.A.C. Potassium leakage test for the evaluation of vigour in soybean seeds. Seed Science and Technology, v.25, n.1, p.7-18, 1996.

DIAS, D.C.F.S.; VIEIRA, A.N.; BHERING, M.C. Condutividade elétrica e lixiviação de potássio para avaliação do vigor de sementes de hortaliças: feijão-devagem e quiabo. Revista Brasileira de Sementes, v.20, n.2, p.408-413, 1998.

DUTRA, A.S.; FILHO, S.M.; TEÓFILO, E.M. Condutividade elétrica em sementes de feijão caupi. Revista Ciência Agronômica, v.37, n.2, p.166-170, 2006.

GOMES, F.P. Curso de estatística experimental. 14.ed., Piracicaba: USP/ESALQ, 2000. 477p.

HAMPTON, J.G.; TEKRONY, D.M. Condutivity test. In:
HAMPTON, J.G.; TEKRONY, D.M. (Ed.). Handbook of vigour test methods. 3.ed. Zürich: ISTA, 1995. p.22-34.

HAMPTON, J.G.; JOHNSTONE, K.A.; EUA-UMPON, V. Bulk conductivity test variables for mungbean, soybean and French bean seed lots. Seed Science and Technology, v.20, n.3, p.677- 686, 1992.

INTERNATIONAL SEED TESTING ASSOCIATION. Seed vigour testing. In: ISTA. International rules for seed testing. Bassersdorf: ISTA, 2004.

KRZYZANOWSKI, F.C.; FRANÇA NETO, J.B.; HENNING, A.A. Relato dos testes de vigor disponíveis para as grandes culturas. Informativo ABRATES, v.1, n.2, p.15-53, 1991.

LOEFFLER, T.M.; TEKRONY, D.M.; EGLI, D.B. The bulk conductivity test as na indicator of soybean seed quality. Journal Seed Technology, v.12, n.1, p.37-53, 1988

MARCOS FILHO, J.; SILVA, W.R.; NOVEMBRE, A.C.; CHAMA, H.C.P.C. Estudo comparativo de métodos para avaliação da qualidade fisiológica de sementes de soja, com ênfase ao teste de condutividade elétrica. Pesquisa Agropecuária Brasileira, v.25, n.12, p.1805-1815, 1990.

MARCOS FILHO, J. Teste de envelhecimento acelerado. In: KRZYZANOWSKI, F.C.; VIEIRA, R.D.; FRANÇA NETO, J.B. (Ed.). Vigor de sementes: conceitos e testes. Londrina: Associação Brasileira de Tecnologia de Sementes, 1999. Cap.3, p.1-24.

MARCOS FILHO, J.; NOVEMBRE, A.D.L.C. Avaliação do potencial fisiológico de sementes de hortaliças. In: NASCIMENTO, W.M. (Ed.). Tecnologia de sementes de hortaliças. Brasília, DF: Embrapa Hortaliças, 2009. p.185246.

McDONALD, M.B. Improving our understanding of vegetable and flower seed quality. Seed Technology, v.20, p.121-124, 1998.

MARTINS, C.C.; MARTINELLI-SENEME, A.; CASTRO, M.M.; NAKAGAWA, J; CAVARIANI, C. Comparação entre métodos para a avaliação do vigor de lotes de sementes de couve-brócolos (Brassica oleracea L. var. italica Plenk). Revista Brasileira de Sementes, v.24, n.2 p.96-101, 2002.

NOVEMBRE, A.D.L.C.; CARPI, V.A.F.; MARCOS FILHO, J.; CHAMMA, H.M.C.P. Teste de condutividade elétrica para estimar o potencial fisiológico de sementes de berinjela. Horticultura Brasileira, v.20, n.2, p.293, 2002.

OLIVEIRA, S.R.S.; NOVEMBRE, A.D.L.C. Teste de 
condutividade elétrica para as sementes de pimentão. Revista Brasileira de Sementes, v.27, n.1, p.31-36, 2005.

OLIVEIRA, M.A.; MATTHEWS, S.; POWELL, A.A. The role of split seed coats in determining seed vigour of commercial seed lots of soybean, as measured by the electrical conductivity test. Seed Science and Technology, v.12, n.2, p.659-668, 1984.

PANOBIANCO, M.; MARCOS FILHO, J. Comparação entre métodos para avaliação da qualidade fisiológica de sementes de pimentão (Capsicum annuum L.) Revista Brasileira de Sementes, v.20, n.2, p.306-310, 1998.

RIBEIRO, D.M.C.A.; CARVALHO, M.L.M.; SALGADO, K.C.C. Avaliação da qualidade fisiológica de sementes de milho através do teste de condutividade elétrica (bulk). Informativo Abrates, v.7, n.1/2, p.187, 1997.

RODO, A.B.; TILLMANN, M.A.A.; VILLELA, F.A.; SAMPAIO, N.V. Teste de condutividade elétrica em sementes de tomate. Revista Brasileira de Sementes, v.20, n.1, p.29-38, 1998.

TAO, J.K. Factors causing variations in the conductivity test for soybean seeds. Journal of Seed Technology, v.3, n.1, p.10-18, 1978.

TICKOO, J.L.; SATYANARAYANA, A. Progress in mung bean breeding research with special emphasis on disease and insect resistance, constraints, and future directions. In: INTERNATIONAL CONSULTATION WORKSHOP ON MUNGBEAN, 1997, New Delhi, Índia. Proceedings... Tainan, Taiwan: AVRDC, 1998. p.58-77.

VANZOLINI, S.; NAKAGAWA, J. Teste de condutividade elétrica em sementes de amendoim. Revista Brasileira de Sementes, v.27, n.2, p.151-158, 2005.

VANZOLINI, S.; NAKAGAWA, J. Teste de condutividade elétrica em sementes de amendoim: períodos de embebição. Informativo ABRATES, v.7, n.1/2, p.169, 1997.

VANZOLINI, S.; NAKAGAWA, J. Teste de condutividade elétrica em sementes de amendoim: efeitos de temperatura e de período de embebição. Revista Brasileira de Sementes, v.21, n.1, p.41-45, 1999a.

VANZOLINI, S.; NAKAGAWA, J. Teste de condutividade elétrica em sementes de amendoim: efeitos de teor de água inicial e de período de embebição. Revista Brasileira de Sementes, v.21, n.1, p.46-52, 1999 b.

VIEIRA, R.D.; PAIVA-AGUERO, J.A.; PERECIN, D.; BITTENCOURT, S.R.M. Correlation of electrical conductivity and other vigor tests with field emergence of soybean seedlings. Seed Science and Technology, v.27, n.1, p.67-75, 1999a.

VIEIRA, R.D.; PAIVA-AGUERO, J.A.; PERECIN, D. Electrical conductivity and field performance of soybean seeds. Seed Technology, v.21, n.1, p.15-24, 1999b.

VIEIRA, R.F.; OLIVEIRA, V.R.; VIEIRA, C. Cultivo do feijão-mungo-verde no verão em Viçosa e em Prudente de Morais. Horticultura Brasileira, v.21, n.1, p.37-43, 2003.

VIEIRA, R.D. Teste de condutividade elétrica. In: VIEIRA, R.D.; CARVALHO, N.M. (Ed.). Testes de vigor em sementes. Jaboticabal: FUNEP, 1994. p.103-132.

VIEIRA, R.D.; KRZYZANOWSKI, F.C. teste de condutividade elétrica. In: KRZYZANOWSKI, F.C.; VIEIRA, R.D.; FRANÇA NETO, J.B. (Ed.). Vigor de sementes: conceitos e testes. Londrina: ABRATES, 1999. cap.4, p.1-26.

VIEIRA, R.D; DUTRA, A.S. Condutividade elétrica em sementes de Abóbora, híbrido Bárbara. Horticultura Brasileira, v.24, n.3, p.305-308, 2006. 